This document is confidential and is proprietary to the American Chemical Society and its authors. Do not copy or disclose without written permission. If you have received this item in error, notify the sender and delete all copies.

\title{
Site-Specific Controlled Growth of Coiled Lambda-Shaped Carbon Nanofibers for Potential Application in Catalyst Support and Nanoelectronics
}

\begin{tabular}{|c|c|}
\hline Journal: & ACS Applied Nano Materials \\
\hline Manuscript ID & an-2020-01374h.R2 \\
\hline Manuscript Type: & Article \\
\hline $\begin{array}{r}\text { Date Submitted by the } \\
\text { Author: }\end{array}$ & $n / a$ \\
\hline Complete List of Authors: & $\begin{array}{l}\text { Lutz, Christian; Karlsruher Institut für Technologie (KIT), Institut für } \\
\text { Mikrostrukturtechnik (INT) } \\
\text { Bog, Uwe; n.able GmbH } \\
\text { Thelen, Richard; Karlsruher Institut fur Technologie - Campus Nord, } \\
\text { Institute of Microstructure Technology } \\
\text { Syurik, Julia; Karlsruher Institut fur Technologie - Campus Nord, } \\
\text { Institute of Microstructure Technology } \\
\text { Malik, Sharali; Karlsruher Institut für Technologie (KIT), Institut für } \\
\text { Nanotechnologie (INT) } \\
\text { Greiner, Christian; Karlsruher Institut für Technologie (KIT), Institut } \\
\text { Angewandte Materialien (IAM) } \\
\text { Hoelscher, Hendrik; Karlsruher Institut fur Technologie - Campus Nord, } \\
\text { Institute of Microstructure Technology } \\
\text { Hirtz, Michael; Karlsruher Institut für Technologie (KIT), Institut für } \\
\text { Nanotechnologie (INT) }\end{array}$ \\
\hline
\end{tabular}

\section{SCHOLARONE" Manuscripts}




\title{
Site-Specific Controlled Growth of Coiled Lambda-
}

\section{Shaped Carbon Nanofibers for Potential Application}

\section{in Catalyst Support and Nanoelectronics}

\author{
Christian Lutz, Uwe Bog, Richard Thelen, Julia Syurik, Sharali Malik, Christian Greiner, \\ Hendrik Hölscher, * and Michael Hirtz*
}

KEYWORDS: branched carbon nanofibers, helical carbon nanofibers, coiled carbon nanofibers, scanning probe lithography

\begin{abstract}
Carbon nanofibers (CNFs), in particular branched ones, raise high interest because of their potential for nanoelectronics, catalyst presentation and applicability as dry adhesives. Here, we present a facile method based on an open ethanol flame in a microchannel for the controlled growth of coiled lambda-shaped carbon nanofibers (c $\lambda \mathrm{CNFs})$. The c $\lambda \mathrm{CNFs}$ consist of two coiled foot CNFs anchored to the substrate and a non-coiled head CNF. The number of twists in the helical structure of the foot CNFs is always of same number and in opposite direction of rotation for a given $c \lambda C N F$. The growth position of the $c \lambda C N F s$ on a substrate can be controlled by targeted deposition of nickel salt via an atomic force microscopy cantilever. An extensive characterization of the $\mathrm{c} \lambda \mathrm{CNFs}$ allows to understand the growth process and to develop a model explaining the observed features of the structures. The presented facile but controlled fabrication process for $\mathrm{c} \lambda \mathrm{CNFs}$ offers a promising route for targeted synthesis of a novel carbon
\end{abstract}


structure with chiral sub-components for experimental and application use as in site-specific growth of branched CNFs for nanoelectronics or local presentation of catalysts.

\section{MAIN TEXT}

Carbon nanotubes and -fibers can be grown in different types and shapes. ${ }^{1-4}$ Branched carbon nanotubes (CNTs), i.e. structures such as Y-shapes, are of high interest due to their potential use in the field of nano-electrical devices. ${ }^{4-6}$ Additionally, Y-shaped CNTs or carbon nanofibers (CNFs) can be used for mimicking hierarchical nanostructures found in nature, such as the nanostructures at the toes of Geckos, ${ }^{7-9}$ enabling their climbing ability. CNTs and CNFs without branches are still used for mimicking the nanostructures of geckos for their use as dry adhesives, ${ }^{10-14}$ but branched CNFs could potentially mimick structures more closely. Finally, the use of CNFs as catalyst support gathered high interest for improvement of catalytic activity. ${ }^{15-18}$ Several approaches were presented to fabricate branched CNTs or CNFs. ${ }^{5,19,28,29,20-27}$ Another peculiar subset of carbon structures are wound-up CNFs/CNTs. These twisted structures are commonly referred to as coiled or helical CNFs/CNTs and are of particular interest for introducing chirality into the system.,30-35 They found applications e.g. as highly efficient adsorbent for wastewater treatment. ${ }^{36}$ While progress has been made on the bulk production of coiled CNFs, $, 3,33,37$ despite the richness of approaches and obtained carbon structures, the growth of single $\mathrm{CNT} / \mathrm{CNF}$ based nanostructures on defined positions and tuning their shape is still a tremendous challenge. In a previous study, we discovered a growths mode for lambda shaped CNFs (labelled $\Lambda \mathrm{CNFs}$ or $\lambda \mathrm{CNFs}$, dependent on their geometry) with two feet anchored to the growing substrate and optional a free standing head. ${ }^{29}$ These nanostructures grow in an open 
ethanol flame from $\mathrm{NiCl}_{2} * 6 \mathrm{H}_{2} \mathrm{O}$ catalyst sites deposited onto a $7 \mathrm{~nm}$ thick Cr-layer on a $\mathrm{SiO}_{2}$ wafer. To reduce the $\mathrm{NiCl}_{2}$-catalysts, it was placed in adjacency to copper bars on the substrate that act as catalyst to provide the required $\mathrm{H}_{2}$ from an ethanol flame. ${ }^{38}$

Here, we present an approach to utilize the open ethanol flame process to grow carbon nanostructures with two coiled "legs" and a non-coiled "head" at defined positions, which we name coiled lambda-shaped CNFs (c $\lambda \mathrm{CNFs}$ ). The detailed characterization of the obtained c $\lambda \mathrm{CNFs}$ allows us to propose a growth model that can explain the unique shape of this fiber structure with only coiled legs but non-coiled head CNFs.

\section{Results and Discussion}

Controlled growth of coiled lambda-shaped CNFs. Commonplace methods to grow CNFs and CNTs are chemical vapor deposition (CVD) ${ }^{39,40}$ or plasma enhanced chemical vapor deposition (PECVD). ${ }^{41}$ However, there are several studies using an open flame process to synthesize CNTs and CNFs as an alternative. ${ }^{13,29,42-49}$ These processes need less infrastructure and benefit from low process costs. Inspired by these studies we used an open ethanol flame to grow coiled lambda-shaped CNFs (c $\lambda \mathrm{CNFs}$ ) from $\mathrm{NiCl}_{2} * 6 \mathrm{H}_{2} \mathrm{O}$ catalysts on a substrate. The substrate consists of a $\left(10 \times 10 \mathrm{~mm}^{2}\right) \mathrm{SiO}_{2}$ wafer with a layer of $7 \mathrm{~nm} \mathrm{Cr}$ on top and copper bars with widths of $14 \mu \mathrm{m}$, heights of $\sim 5 \mu \mathrm{m}$ and a periodicity of $\sim 100 \mu \mathrm{m}$. Using the tip of an atomic force microscope cantilever, ${ }^{50,51}$ the catalyst size and position on the substrate where CNFs will grow, can be defined with high precision. ${ }^{29}$ To obtain the c $\lambda \mathrm{CNFs}$, catalytic salt $\left(\mathrm{NiCl}_{2} * 6 \mathrm{H}_{2} \mathrm{O}\right)$ was deposited on the substrate between the copper bars (Figure 1a)). The critical volume of $\mathrm{NiCl}_{2} * 6 \mathrm{H}_{2} \mathrm{O}$ to grow single lambda-shaped CNFs was determined in our previous study to $0.033 \mu \mathrm{m}^{3}$ (Figure S1). ${ }^{29}$ Too high temperatures and humidities during the preparation process of 
the sample might lead to oxidization of the $\mathrm{NiCl}_{2} * 6 \mathrm{H}_{2} \mathrm{O}$ catalysts preventing CNF growth. ${ }^{13,52}$ Therefore, the samples were prepared at lab temperatures below $23{ }^{\circ} \mathrm{C}$ and in relative humidities below $50 \%$. Additionally, the samples were dried for more than $24 \mathrm{~h}$ before use, to reduce residual humidity to a minimum.

a)

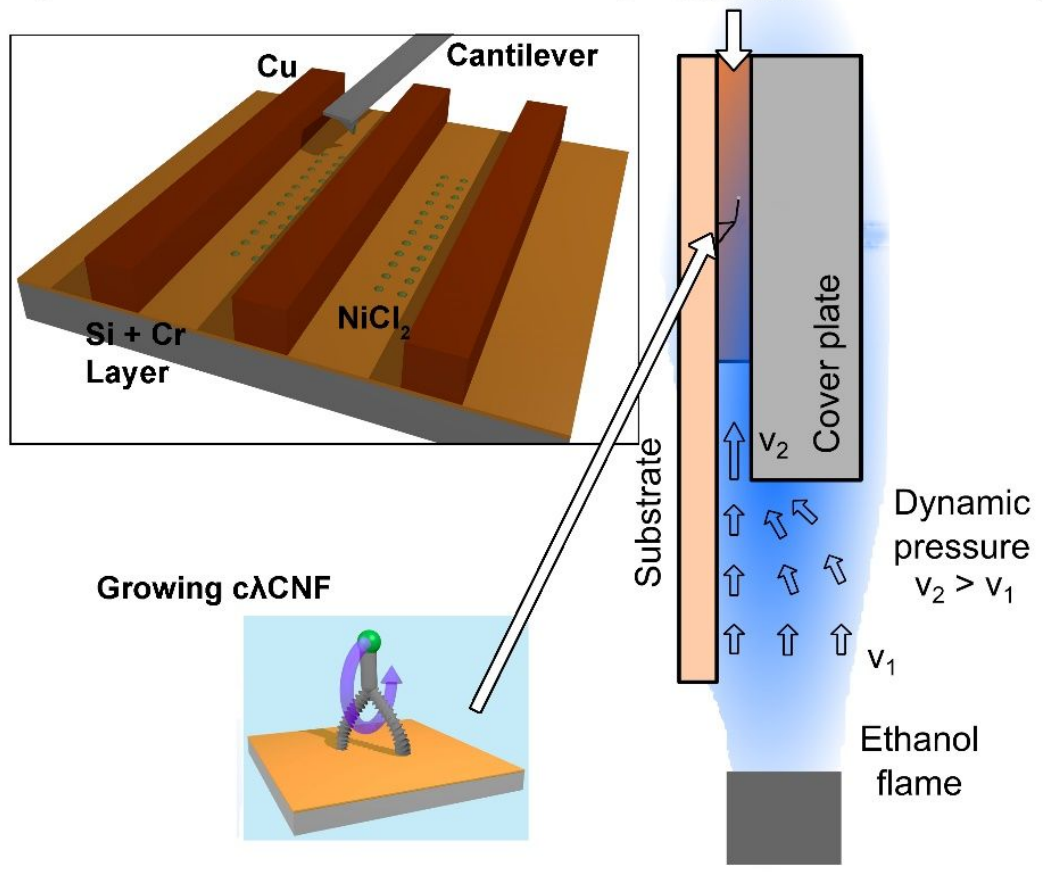

c)

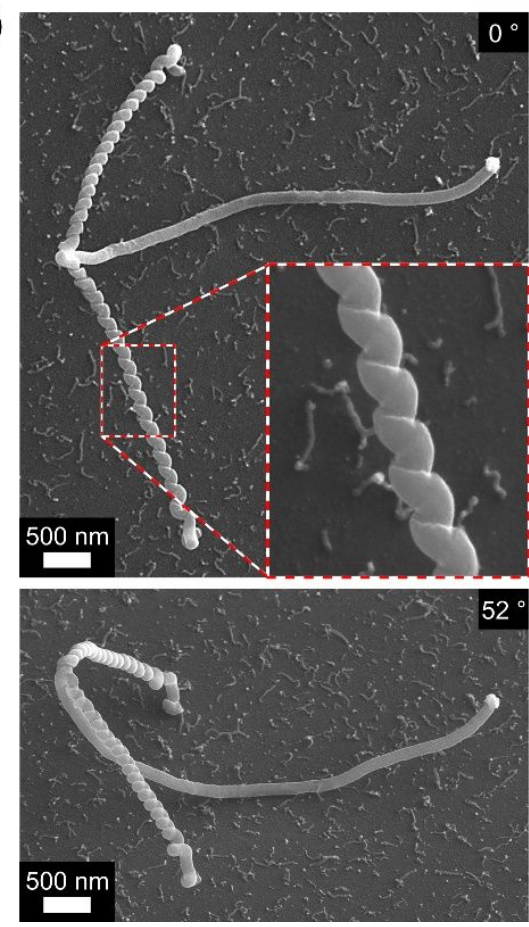

Figure 1. Growing of coiled lambda-shaped CNFs. (a) Schematic of the atomic force microscope cantilever based writing process to deposit the catalytic salt $(\mathrm{NiCl} 2 * 6 \mathrm{H} 2 \mathrm{O})$ on the substrate between the copper bars. (b) Schematic setup with the microchannel in the open ethanol flame for the growth of coiled lambda-shaped CNFs. (c) During ethanol flame synthesis coiled lambda-shaped CNFs grow from the deposited catalysts.

During growth, hydrogen is produced from the copper in the ethanol flame, which is required to reduce Ni-oxide possibly formed from the $\mathrm{Ni}$-salt catalyst to a pure state. ${ }^{38}$ To achieve a stable 
ethanol flame without flicker we used a setup based on a closed system with guided air inlet and outlet as described previously. ${ }^{29}$ On the position of the catalysts spotted in between the copper bars, the sample was covered with an $\mathrm{Al}_{2} \mathrm{O}_{3}$-plate, forming channel structures with a width of $\sim 100 \mu \mathrm{m}$ and a height up to $\sim 10 \mu \mathrm{m}$. The such prepared sample was then positioned vertically aligned in the ethanol flame, at a height of $2 \mathrm{~mm}$ over the wick of the ethanol burner (Figure 1b)). The ethanol flame temperature at the position, where c $\lambda C N F$ s grow, was measured with a thermocouple to $750{ }^{\circ} \mathrm{C}$. The growth time was 5 minutes for all experiments and a typical outcome is shown in Figure 1c. The obtained CNF structures have two coiled feet anchored to the substrate and a non-coiled head on top. Due to their appearance, we named these structures coiled lambda-shaped CNFs, abbreviated as c $\lambda \mathrm{CNFs}$.

Geometry analysis of coiled lambda-shaped CNFs. Our geometry analysis shows that the c $\lambda \mathrm{CNFs}$ have diameters in the range of $200 \mathrm{~nm}$, approximately half of the diameters of previously grown non-coiled lambda-shaped CNFs. ${ }^{29}$ However, the lengths of the three parts (two legs and one head) can be much larger compared to the conventional lambda-shaped CNFs. The head CNF can reach lengths over $5 \mu \mathrm{m}$ and the distance between the positions where the two leg CNFs are anchored to the substrate is up to $10 \mu \mathrm{m}$. This can be explained with a higher flow velocity in the microchannel structures, transporting more carbon from the ethanol flame to the CNFs and leading to a higher growth rate. 
a)

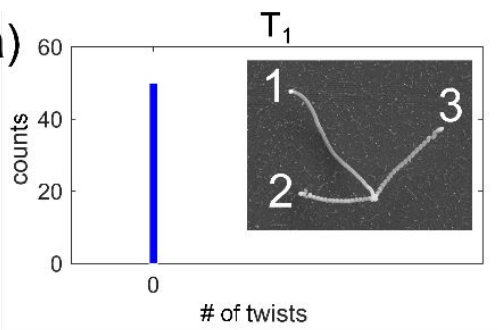

b)

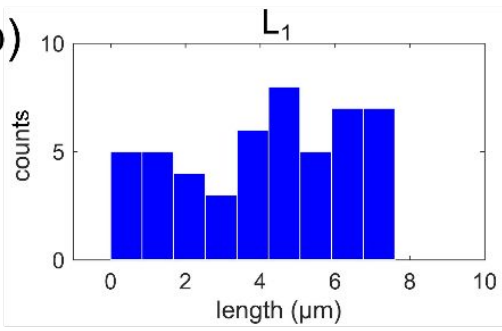

C)

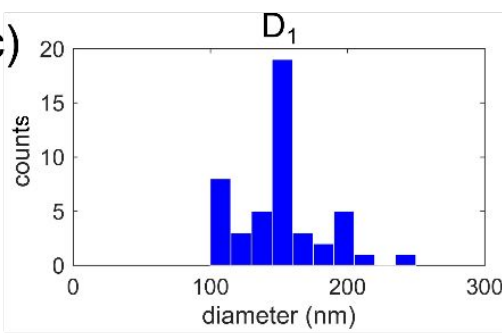

d)

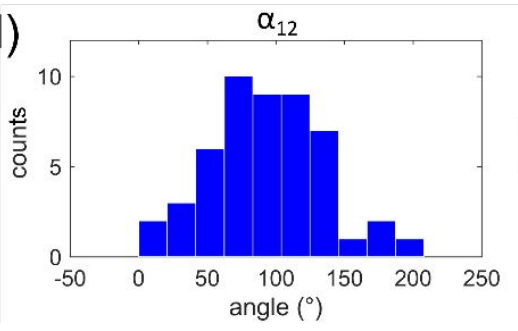

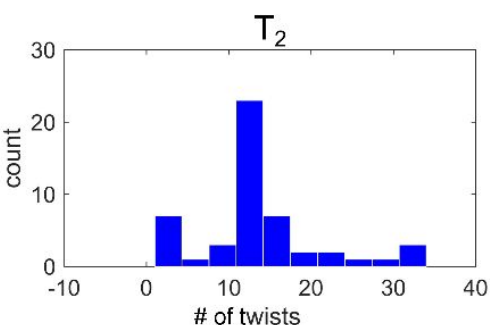

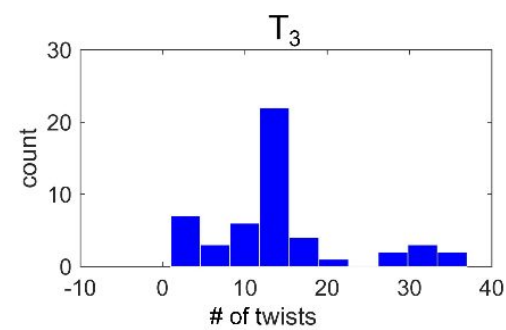

$\mathrm{L}_{2}$
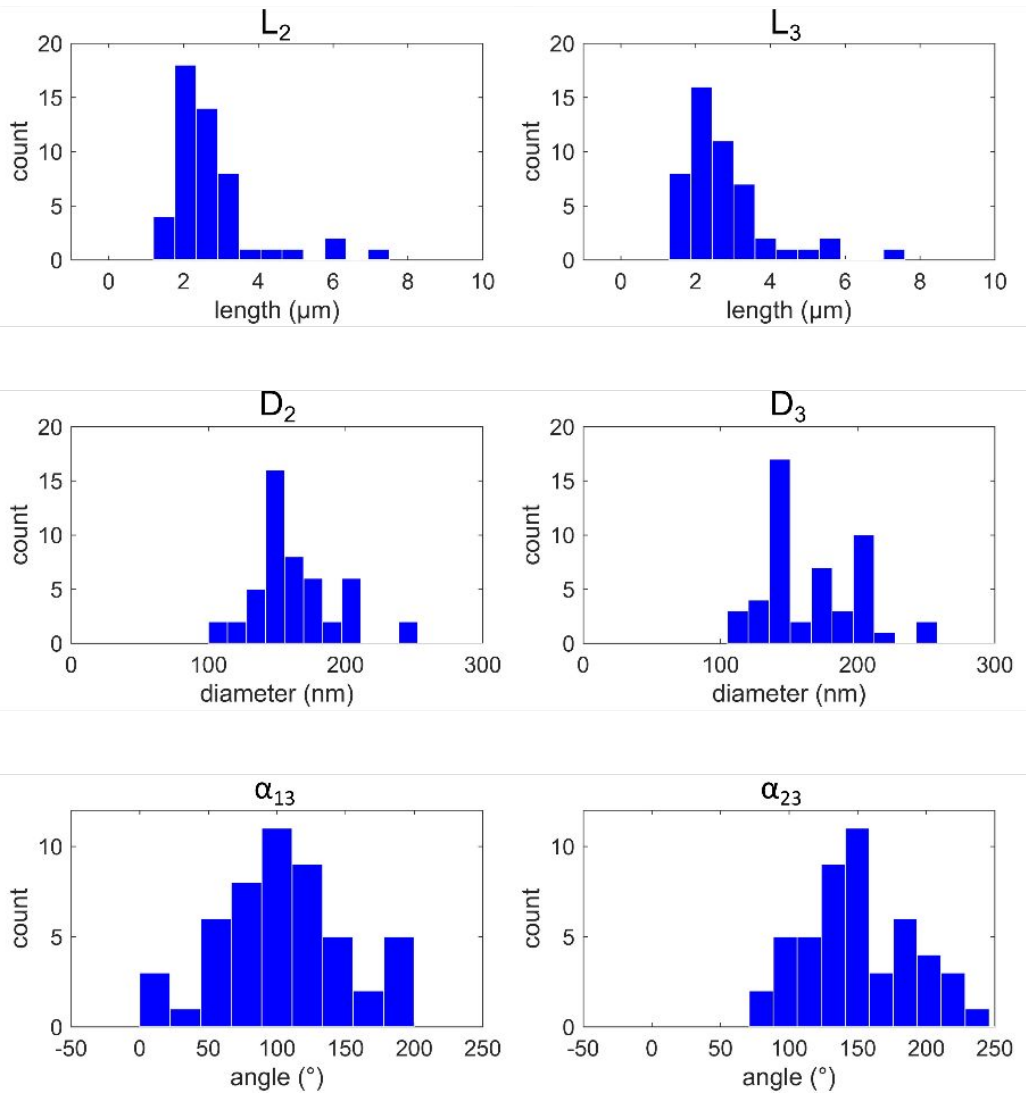

Figure 2. Geometry analysis of 50 c $\lambda$ CNFs. The head part is indicated with " 1 ", the foot counterclockwise from the head in the top view is " 2 " and the other foot is " 3 " (as depicted in the first diagram). The diagrams represent: (a) the number of twists, (b) the lengths, (c) the diameters and $(\mathrm{d})$ the angles between the three CNF parts.

For an exact geometry analysis, $50 \mathrm{c} \lambda \mathrm{CNFs}$ where imaged by SEM. The head part is indicated with " 1 ", the foot counterclockwise from the head in the top view is " 2 " and the other foot is " 3 ". 
The summarized results for the c $\lambda \mathrm{CNF}$ s geometry is given in Figure 2. The number of twists of the two CNFs anchored to the substrate are $\mathrm{T}_{2}=14.0 \pm 7.5$ ( $N=50$ here and in the following) and $\mathrm{T}_{3}=14.2 \pm 8.3$ and are nearly identical, whereas no twists were observed for the free-standing head CNF in any cases. The lengths of the two CNFs connected to the substrate surface are $\mathrm{L}_{2}=(2.8 \pm 1.2) \mu \mathrm{m}$ and $\mathrm{L}_{3}=(2.8 \pm 1.2) \mu \mathrm{m}$ and are identical, whereas the free-standing head $\mathrm{CNF}$ can be much longer $\mathrm{L}_{1}=(4.2 \pm 2.3) \mu \mathrm{m}$. The diameters of the two CNFs connected to the substrate surface are $D_{2}=(152.2 \pm 32.7) n \mathrm{~nm}$ and $\mathrm{D}_{3}=(166.3 \pm 39.7) \mathrm{nm}$ and are slightly smaller as the diameter of the free-standing head $C N F D_{1}=(171.0 \pm 42.1) \mathrm{nm}$. The angles between the two CNFs connected with the substrate are $\alpha_{23}=(154.8 \pm 53.9)^{\circ}$, whereas the other two angles between the head CNF and the two leg CNFs are smaller with $\alpha_{12}=(94.87 \pm 43.5)^{\circ}$ and $\alpha_{13}=(105.0 \pm 48.5)^{\circ}$. 


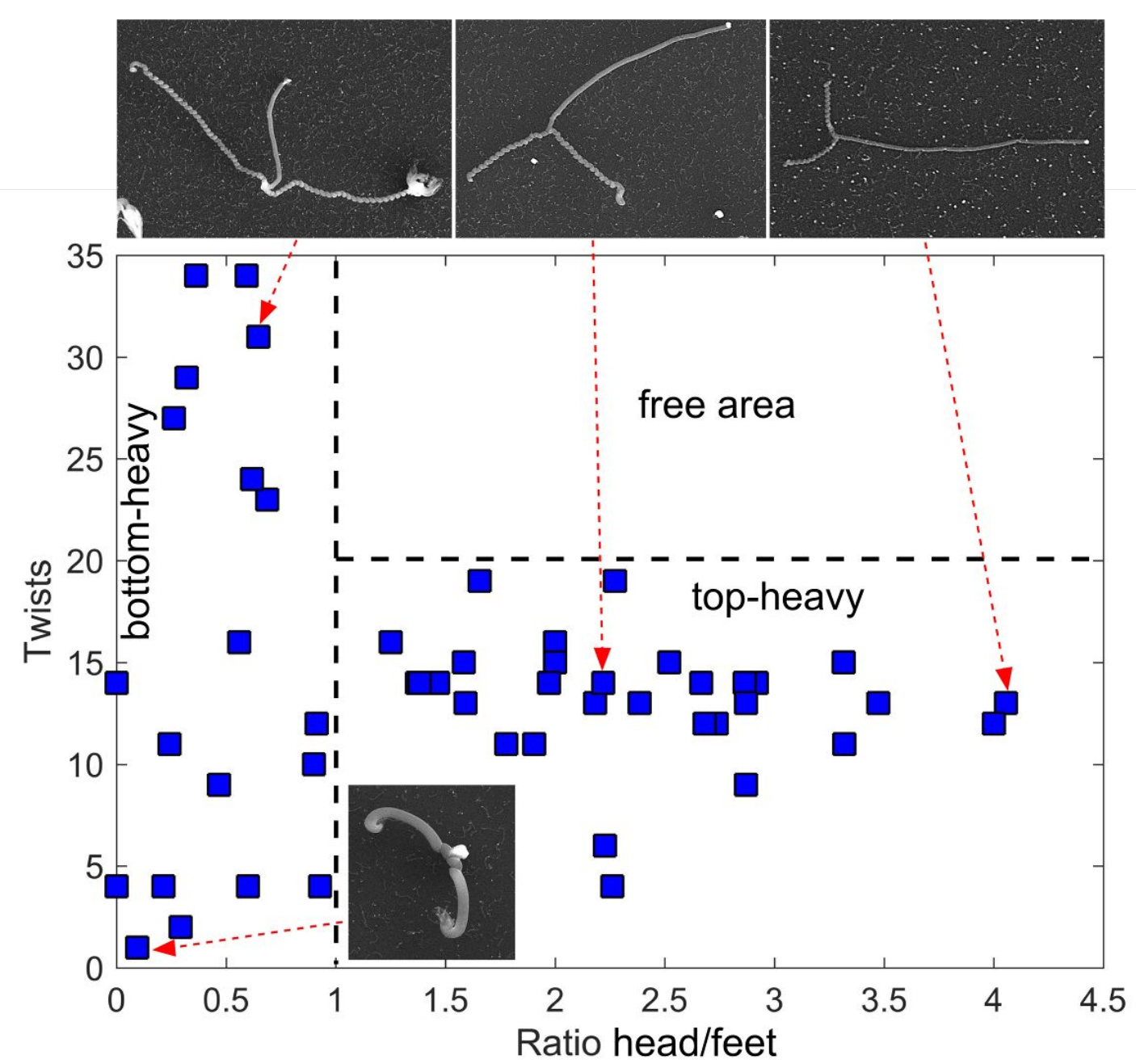

Figure 3. Diagram of the number of feet twists as a function of the ratio of head and feet CNF length. The data points represent $50 \mathrm{c} \lambda \mathrm{CNFs}$ grown in a 5 minutes process. The insets exemplify the morphology of selected $\mathrm{c} \lambda \mathrm{CNF}$. Their respective data point in the diagram is indicated by arrows.

In order to group the $\mathrm{c} \lambda \mathrm{CNFs}$ data for further analysis, we plotted the head/feet length ratio and the number of twists in the CNF feet in a diagram (Figure 3) showing the number of twists vs. the length ratio between head and feet. A great range of different values indicate that the c $\lambda$ CNFs nucleate at different points in time during the overall growth period of 5 minutes. We 
observed a free area above a ratio of 1.0 and 20 twists (which is no artifact of the specific growth time, but can be understood in terms of the c $\lambda$ CNF growth model discussed in the next section). The diagram also reveals that $\mathrm{c} \lambda \mathrm{CNFs}$ can develop to bottom-heavy or top-heavy configurations.

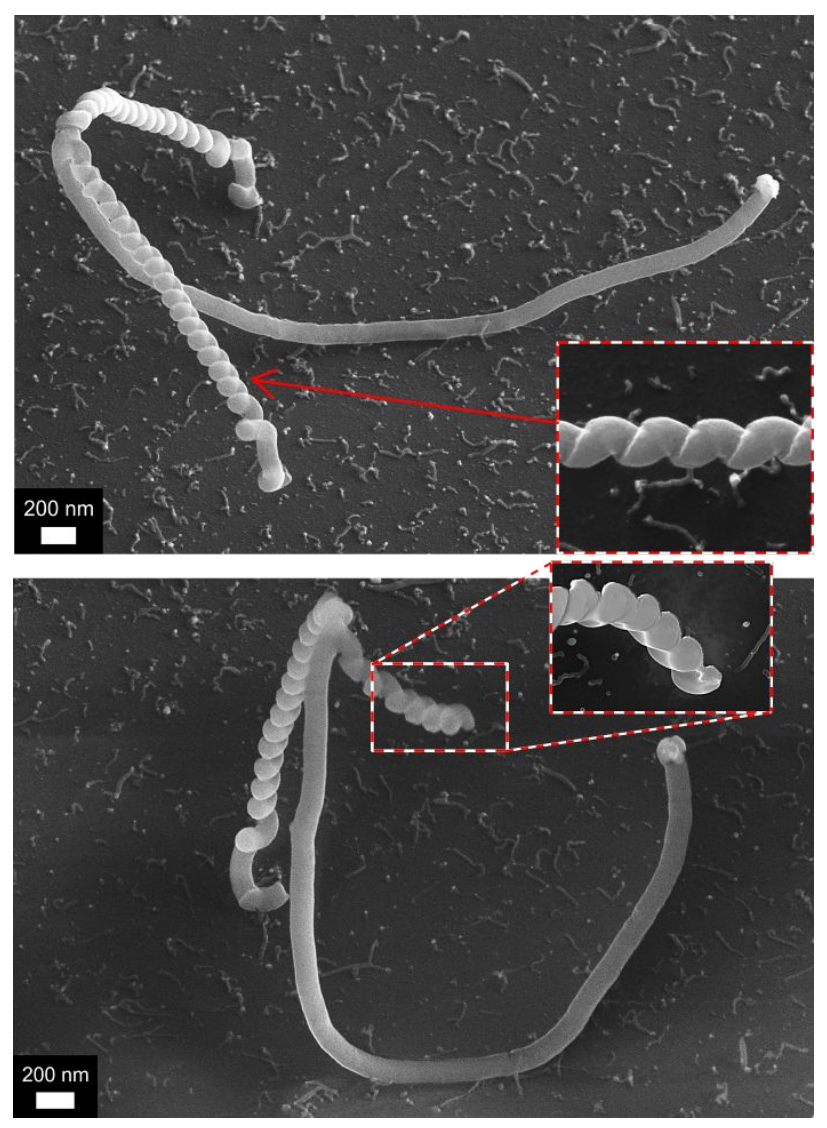

Figure 4. A c $\lambda \mathrm{CNF}$ with one CNF foot cut off the substrate using a focused ion beam. While the overall shape of the structure changed due to release of mechanical stress, the twists in the feet CNFs remained unaltered.

To probe its mechanical stability, one leg CNF of a c $\lambda \mathrm{CNF}$ was cut utilizing a focused ion beam (FIB). Figure 4 shows the respective SEM images of the c $\lambda$ CNF before (top) and after the cut (bottom). After cutting, the complete structure moved and changed shape, which is most 
likely caused due to relaxation of internal stress in the structure after the cut. This outcome is different to our previous study where we grew non-coiled $\lambda \mathrm{CNF}$ which showed no obvious relaxation by immediate structural change. ${ }^{29}$ However, the twists by themselves remain unchanged, implicating that they are fixated into the structure after removing the sample from the ethanol flame.

In addition to the geometrical analysis, also the chemical composition of the c $\lambda$ CNFs was explored by Energy-Dispersive X-ray spectroscopy (EDS), results are shown in Table 1.

Table 1. EDS measurement of $c \lambda C N F$. The $w t \%$ and standard deviation of the elemental composition was determined by the AZtec software.

\begin{tabular}{lccccc}
\hline Position & $\mathrm{C}[\mathrm{wt} \%]$ & $\mathrm{O}[\mathrm{wt} \%]$ & $\mathrm{Si}[\mathrm{wt} \%]$ & $\mathrm{Cu}[\mathrm{wt} \%]$ & $\mathrm{Ni}[\mathrm{wt} \%]$ \\
\hline $\begin{array}{l}\text { CNF } \\
\text { neck-part }\end{array}$ & $25.29 \pm 0.46$ & $34.95 \pm 0.31$ & $39.73 \pm 0.29$ & Not detected & Not detected \\
$\begin{array}{l}\text { CNF } \\
\text { twisted-foot- } \\
\text { part }\end{array}$ & $27.85 \pm 0.46$ & $34.68 \pm 0.32$ & $37.42 \pm 0.28$ & Not detected & Not detected \\
$\begin{array}{l}\text { Catalytic } \\
\text { center }\end{array}$ & $9.69 \pm 0.49$ & $39.69 \pm 0.32$ & $46.61 \pm 0.33$ & $3.44 \pm 0.13$ & $0.56 \pm 0.07$ \\
$\begin{array}{l}\text { Reference } \\
\text { (free-area) }\end{array}$ & $8.49 \pm 0.49$ & $38.10 \pm 0.32$ & $53.33 \pm 0.36$ & Not detected & Not detected \\
\hline
\end{tabular}

A substrate area free of CNFs was chosen as reference area, showing a clear signal of silicon oxide $\left(\mathrm{SiO}_{2}\right)$ in EDX, as well as the background levels of the other analyzed materials. The neckpart and the coiled foot-part of the c $\lambda \mathrm{CNF}$ have nearly the same material composition of carbon and residual amounts of oxygen, silicon, copper and nickel. The apparent low amount of carbon is an artifact resulting from the high background levels of silicon and oxygen from the substrate. The graphitic carbon nature of the CNFs is clearly confirmed by the Raman spectra (Figure 5), as 
was expected from previous studies of CNF growth in the open ethanol flame process. ${ }^{13}$ The catalytic centers appear to consist of a nickel/copper alloy (1:6).

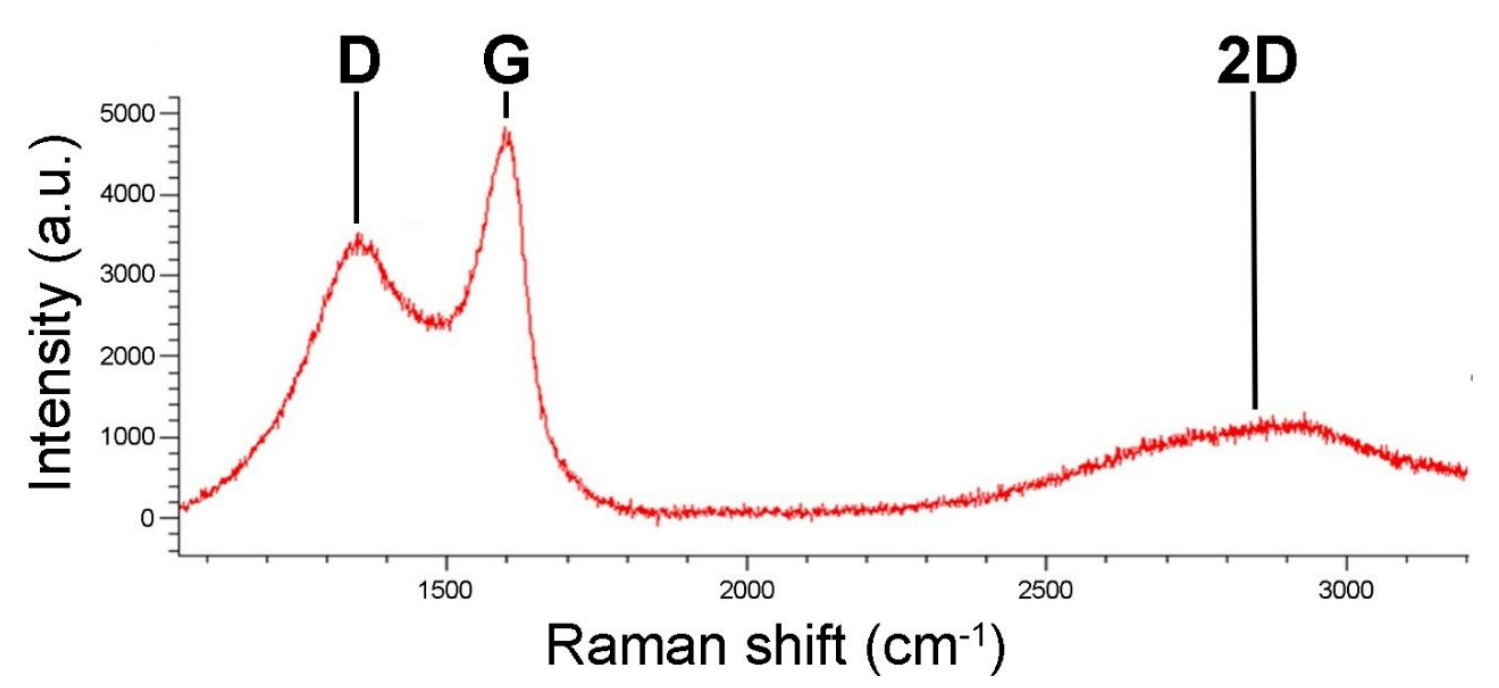

Figure 5. Raman spectra of coiled lambda-shaped CNFs at $514 \mathrm{~nm}$, showing a sharp G band which is indicative of graphitic CNFs, further confirmed by the $\mathrm{I}_{\mathrm{D}} / \mathrm{I}_{\mathrm{G}}$ ratio of 0.736 . A broadened $2 \mathrm{D}$ band (attributed to strain) ${ }^{53}$ is observed.

Coiled lambda-shaped CNF Growth Mechanism. During the last decades, growth of coiled CNFs and CNTs was reported by several groups and different growths models were suggested. Nonetheless, although early reports on vermicular CNFs date back to $1954,{ }^{54}$ no definitive or unified growth model has emerged yet. Hence, it is no surprise that most reports on coiled CNFs/CNTs fully abstain on suggesting a growth model. The diverse range of circumstances in regard to position of catalytic center during growth, pairwise or singular CNF/CNT growth, used techniques and process parameters, and morphology in obtained CNFs/CNTs suggest that probably not all coiling in CNFs/CNTs is caused by the same mechanism. Most studies propose either chemical modification during the growth process (introduction of pentagons and 
heptagons into the hexagonal tube lattice $)^{55-58}$ and/or the unequal extrusion of carbon material from the catalytic center, ${ }^{59-62}$ but mechanical causes $^{63}$ and thermodynamic/entropical reasons ${ }^{58,64}$ were hypothesized, too. In regard to possible growth mechanisms for our c $\lambda \mathrm{CNFs}$, two specific morphologies strike out as bearing significant resemblance: $\mathrm{X} / \mathrm{Y}$ junction carbon nanocoils ${ }^{28}$ and carbon coils growing pairwise from a single catalytic center. ${ }^{35,63,65,66}$ For the coiled carbon structures with $\mathrm{X}$ and $\mathrm{Y}$ junctions, a growth mechanism based on the either merging of independently growing carbon coils or growth of three or even four carbon coils from a matching number of facets on the catalytic center was suggested..$^{28}$ This cannot explain the growth of the c $\lambda \mathrm{CNFs}$, as these structures always originate from a single catalytic center (as observed in the general $\lambda \mathrm{CNFs}$ ). ${ }^{29}$ Furthermore, the consistent switch from coiled feet CNFs to non-coiled head CNF in each and every observed c $\lambda$ CNFs would not be understood in this growth model.

The pairwise growing carbon coils bear a striking resemblance to the feet CNFs of the

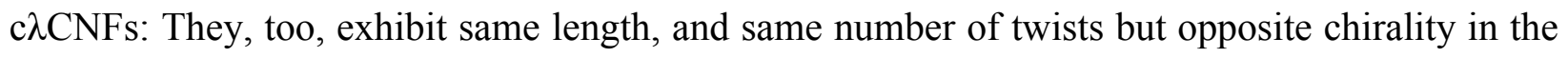
respective pairwise grown carbon coils. ${ }^{35,63,65,66}$ For these, a mechanical origin of the coiling by stress release in the previously straight grown CNFs by a change in van der Waals force mediated attachment to the substrate on temperature variation in the growths process is suggested. ${ }^{63}$ These hypothesis fails for our c $\lambda \mathrm{CNFs}$, too, as we clearly observe the formation of non-coiled $\lambda$ CNFs (that are already only attached to the substrate exclusively at the end points of the feet CNFs) and our process parameters are kept constant during the whole growth process. Interestingly, for the pairwise grown carbon coils, Tang et al. reported a preferred angle between the two carbon coils of $70^{\circ}$ with a minority of pairs with $35^{\circ}$ or $130^{\circ}$, respectively, but no intermediate angles ${ }^{65}$ while in our present study we observe a wide range of angles with a mean of $(154.8 \pm 53.9)^{\circ}$ (as described in section 2.2.). This underlines another difference in growth, as 

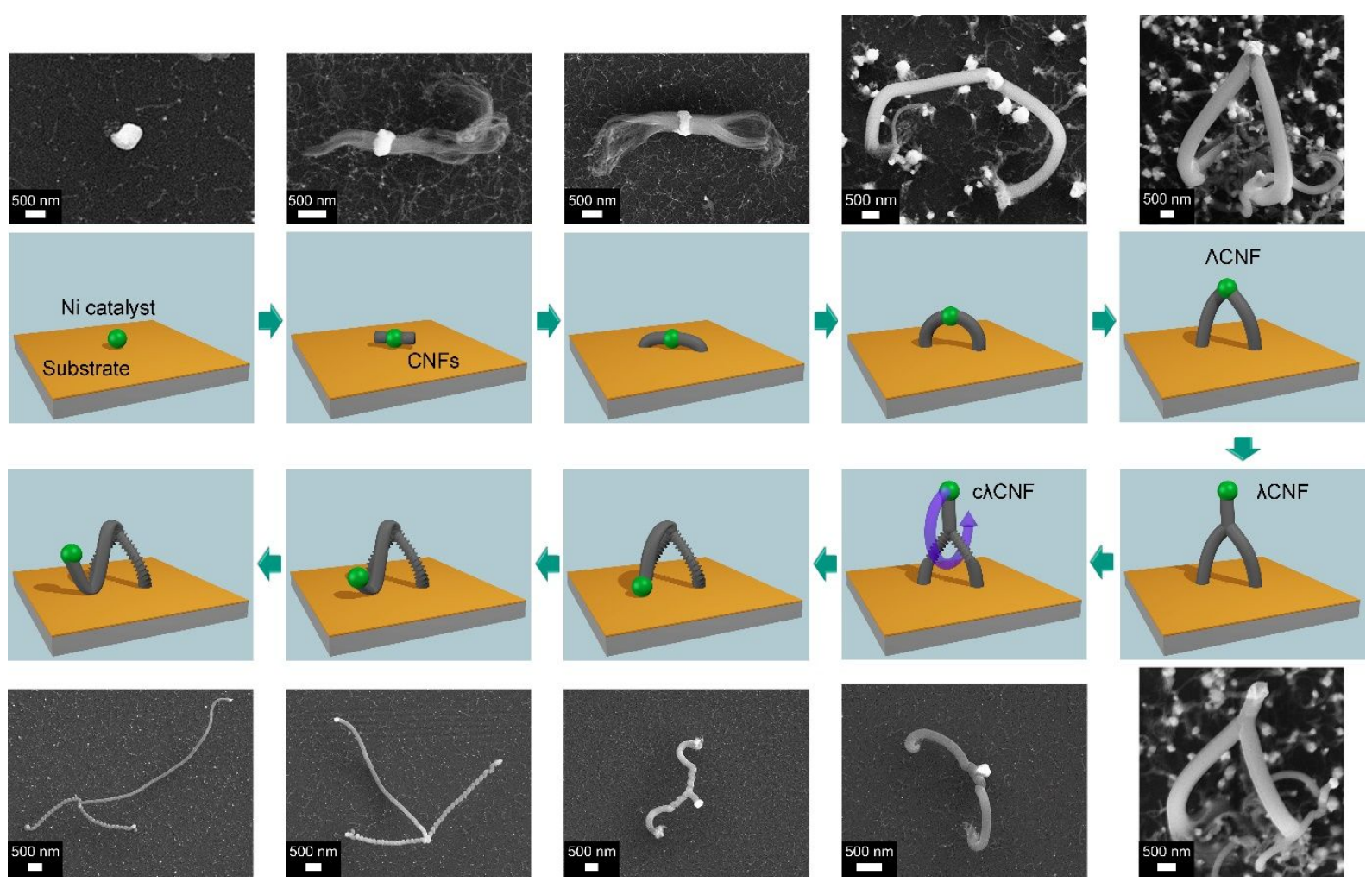

Figure 6. Growth model for c $\lambda \mathrm{CNF}$. Two CNFs grow from one catalytic center made by $\mathrm{Ni}$ forming a $\Lambda \mathrm{CNF}$ and finally a $\lambda \mathrm{CNF}$ as described previously. ${ }^{29}$ After this point is reached, due to the higher flame velocity in the microchannels, the CNF head part starts to rotate through their foot CNFs, winding them into equal number of twists. The head CNF will grow further, until it cannot pass any more through the space between the foot CNFs, thus stopping the winding process. Even then, the head CNF can continue growing, but no additional twists will be introduced to the foot CNFs. Part of the figure is adapted with permission from Ref. ${ }^{29}$.

However, the detailed observation of c $\lambda$ CNFs in different stages of growth allows us to come forward with a possible growth model for our case (Figure 6). As shown in our last work, ${ }^{29}$ for 
non-coiled CNFs, two CNFs grow from one Ni catalytic center forming a $\Lambda$ CNF first and then a $\lambda \mathrm{CNF}$ on further growth. In the present study, we observe a coil structure in the feet CNFs (anchored to the substrate), which we propose to be caused by the CNF head part of $\lambda$ CNFs starting to rotate through their feet $\mathrm{CNF}$, due to the higher flame velocity in the microchannel. This results in twists in the foot CNFs, while the head CNF remains straight. The head CNF will continue to grow during rotation, until reaching a certain length, where rotating is no longer possible for geometrical constraints. After this length is reached, the head CNF can still continue to grow, but the foot CNFs reach their final number of twists.

Having formulated this hypothesis for the growth process, we can discuss our empirical findings on the c $\lambda \mathrm{CNF}$ in light of this model. First, if this hypothesis for the growth process is correct, the number of twists in each of the two legs of the c $\lambda \mathrm{CNF}$ should be equal and independent of their sizes, which was observed in our experiments (Figure 2a)). Secondly, the winding up of the feet CNFs will start up from the Y-junction where the feet and head CNFs meet and not be present prior to emergence of the head CNF. This can also clearly be seen in the panels of Figure 6 directly following the c $\lambda \mathrm{CNF}$, were more and more twists wind up until the whole feet CNFs are coiled. Thirdly, when looking at the length ratio of the head CNFs to the foot CNFs, there should be a clear distinction between top-heavy (ratio $>1.0$, head longer then feet) and bottom-heavy (ratio $<1.0$, feet longer than head) in the number of twists in the foot CNFs: $c \lambda C N F$ with a ratio below 1.0 can rotate much more often through their feet than those with a ratio over 1.0 with the same growth time (Figure 3 ). This data also clearly shows that there is a cut-off of about 20 twists per foot CNF that is not surpassed for top-heavy c $\lambda \mathrm{CNF}$, indicating the stop of twisting at a certain head CNF length in regard to the foot CNFs as predicted by the growth model. The observed "free area" in the diagram is thus also no artefact 
of growth time, as additional growth will result in longer head CNFs without addition of further twists, thus in points farther to the right in the diagram, but not farther up.

a)

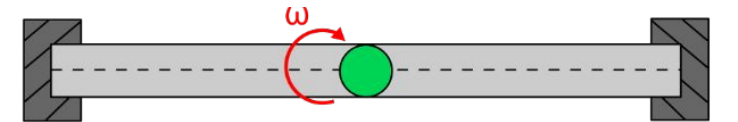

Rotation:

Another deduction from the presented growth model is, that the twists in both of the foot CNFs should be always of opposite direction of rotation (Figure 7a). This opposite direction of rotation

Figure 7. Relative angles in relation to the CNF main axis (angles of twist). a) Sketch of the twisting behavior of a cylinder as model for the foot CNFs of a c $\lambda \mathrm{CNF}$. The rotational direction in each foot CNF must be opposite. b) The relative angle against the main axis as obtained from 50 c $\lambda$ CNF.

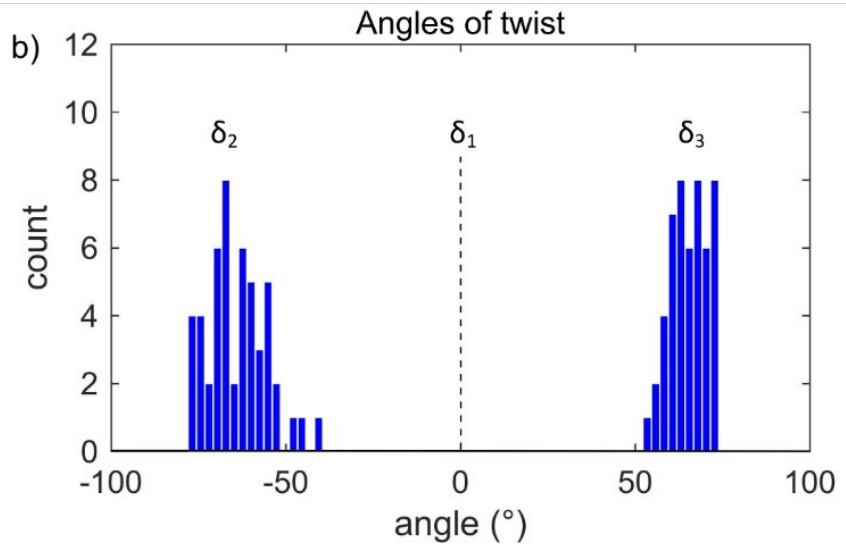




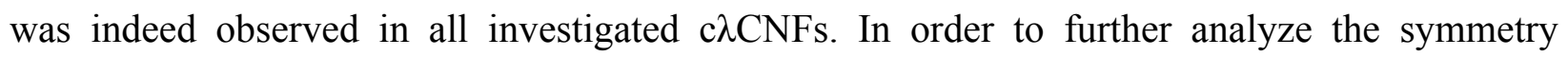
between the respective foot CNFs of a c $\lambda \mathrm{CNF}$ one can also look at the angle of the twists in relation to the CNFs main axis. This angle is generally between $0^{\circ}$ and $90^{\circ}$, as defined in Figure 7a. A tabulation of the measured angles in 50 c $\lambda \mathrm{CNF}$ shows a striking symmetry (Figure 7b). The angles against the main axis (or twisting angle) are for the non-coiled head CNF $\delta_{1}=(0.0 \pm 0.0)^{\circ}(N=50)$, whereas for the two foot CNFs values of $\delta_{2}=(-63.9 \pm 8.5)^{\circ}$ and $\delta_{3}=(65.5 \pm 5.5)^{\circ}$ are obtained.

An alternative hypothesis for the stop of twisting during the growth process is that the twisting introduces a reset force that makes it harder to introduce additional twists. The higher the relative stiffness of the CNF the earlier the twisting would stop. As both foot CNFs are fixed against the substrate, the twisting would stop at the point where the longitudinal stress created by the twisting is equal to the force (introduced to the head CNF by the gas stream of the flame) that causes the twisting during the CNF growth. Here, the free area in the diagram of twists versus head to feet CNFs length ratio (Figure 3) suggests that our proposed geometrical stop of twisting sets in much earlier than any stop by reset force, as for the top-heavy c $\lambda$ CNFs no foot CNFs with more than 20 twists are observed, while the bottom-heavy c $\lambda \mathrm{CNF}$ can have many more twists (up to 34 observed in our sample).

\section{Conclusion}

In summary, we presented a method for the controlled growth of a unique form of coiled lambda-shaped CNFs (c $\lambda \mathrm{CNFs}$ ) in a microchannel placed into an open ethanol flame. The resulting carbonic structures feature two coiled foot CNFs anchored to the substrate and a noncoiled head CNF. The growth position of the cגCNFs can be determined by the controlled 


\section{Methods}

Substrate and microchannel fabrication. For the growth of coiled lambda-shaped CNF, a substrate ( $\mathrm{Si}$ with $7 \mathrm{~nm} \mathrm{Cr}$ on top) with arrays of copper grids was fabricated as described elsewhere. ${ }^{29} \mathrm{~A}$ cover plate made by $\mathrm{Al}_{2} \mathrm{O}_{3}$ was placed over the copper bars to achieve several microchannels. The microchannels in the cross section have a height of 5-10 $\mu \mathrm{m}$ and a length of $60 \mu \mathrm{m}$.

Deposition of catalysts. The cantilever based deposition of catalyst was performed with a commercial system (Molecular Printer, n.able GmbH, Germany). A self-built holder was equipped with a cantilever type 'A' (Nanoink Inc., USA), previously dip-coated with a solution ( $2 \mathrm{mg} / \mathrm{mL}$ ) of $\mathrm{NiCl}_{2} * 6 \mathrm{H}_{2} \mathrm{O}$ in ethanol mixed with glycerol (1:10) as the ink. Deposition was 
performed directly onto the substrate between two copper bars. The deposition was conducted with a dwell time of $4 \mathrm{~s}$ at $60 \%$ relative humidity.

Growth of coiled lambda-shaped CNFs. A self-built machine was used to grow coiled lambda-shaped CNFs, described in detail elsewhere. ${ }^{29,67}$ It consists of an ethanol burner with a combustion rate of $0.4 \mathrm{~mL} / \mathrm{min}$ in a closed system with a guided air flow, to achieve an ethanol flame without jitter. The substrate $\left(10 \times 10 \mathrm{~mm}^{2}\right)$ with the cover plate to grow coiled lambdashaped CNFs was placed vertically in the ethanol flame.

CNF characterization. The morphology of the c $\lambda C N F s$ was investigated with a scanning electron microscopy (SEM) SUPRA 60 VP (Zeiss, Germany). Raman spectra were measured on an inVia Raman microscope (Renishaw, UK) at $514 \mathrm{~nm}$. EDS measurements were performed with a Zeiss Leo 1530 SEM operating at 20kV. The EDS were acquired using the "Point \& ID" option in AZtec software using an Oxford $\mathrm{X}-\mathrm{Max}^{\mathrm{N}} 50$ detector. The detector offers 50 large area silicon drift detectors, which can quantify elements heavier than carbon to an accuracy of $0.01 \%$ by weight, thus the Oxford Instruments AZtec materials characterization system can gather highly accurate data at the micro- and nanoscales. The detector conforms to ISO 15632:2012 Microbeam analysis - Selected instrumental performance parameters for the specification and checking of energy-dispersive X-ray spectrometers for use in electron probe microanalysis.

CNF geometry analysis. For $50 \mathrm{c} \lambda \mathrm{CNF}$ from one experiment with 5 minutes growth time, detailed measurements for length, diameter, angle between the three CNFs and number of coils in the foot CNFs were manually obtained with the onboard SEM software. All reported values are means $\pm \mathrm{SD}$. 
FIB cutting of CNFs. A c $\lambda$ CNF was cut using a focused ion beam setup (Helios Nanolab 650 from FEI, USA) operating at an acceleration voltage of $30 \mathrm{kV}$ with an ion beam current of $80 \mathrm{pA}$. The cutting time was $1 \mathrm{~s}$ for the cut of one foot of the c $\lambda \mathrm{CNF}$.

\section{ASSOCIATED CONTENT}

\section{Supporting Information}

The Supporting Information is available free of charge at

https://pubs.acs.org/doi/10.1021/acsXXXXXX.

Optical micrograph and AFM images of a catalyst array as deposited (PDF)

\section{AUTHOR INFORMATION \\ Corresponding Authors}

Hendrik Hölscher - Institute of Microstructure Technology (IMT) and Karlsruhe Nano Micro Facility (KNMF), Karlsruhe Institute of Technology (KIT), Hermann-von-Helmholtz-Platz 1, 76344 Eggenstein-Leopoldshafen, Germany; Email: hendrik.hoelscher@kit.edu

Michael Hirtz - Institute of Nanotechnology (INT) and Karlsruhe Nano Micro Facility (KNMF), Karlsruhe Institute of Technology (KIT), Hermann-von-Helmholtz-Platz 1, 76344 EggensteinLeopoldshafen, Germany; https://orcid.org/0000-0002-2647-5317; E-mail:

michael.hirtz@kit.edu

\section{Authors}


Christian Lutz - Institute of Microstructure Technology (IMT) and Karlsruhe Nano Micro

Facility (KNMF), Karlsruhe Institute of Technology (KIT), Hermann-von-Helmholtz-Platz 1, 76344 Eggenstein-Leopoldshafen, Germany

Uwe Bog - n.able GmbH, Hermann-von-Helmholtz Platz 1, 76344 Eggenstein-Leopoldshafen, Germany

Richard Thelen - Institute of Microstructure Technology (IMT) and Karlsruhe Nano Micro Facility (KNMF), Karlsruhe Institute of Technology (KIT), Hermann-von-Helmholtz-Platz 1, 76344 Eggenstein-Leopoldshafen, Germany

Julia Syurik - Institute of Microstructure Technology (IMT) and Karlsruhe Nano Micro Facility (KNMF), Karlsruhe Institute of Technology (KIT), Hermann-von-Helmholtz-Platz 1, 76344

Eggenstein-Leopoldshafen, Germany

Sharali Malik - Institute of Quantum Materials and Technology (IQMT), Karlsruhe Institute of Technology (KIT), Hermann-von-Helmholtz-Platz 1, 76344 Eggenstein-Leopoldshafen, Germany

Christian Greiner - Institute for Applied Materials (IAM) and Karlsruhe Nano Micro Facility (KNMF), Karlsruhe Institute of Technology (KIT), Hermann-von-Helmholz-Platz 1, 76344 Eggenstein-Leopoldshafen, Germany and KIT IAM-CMS MikroTribologie Centrum $\mu$ TC, Strasse am Forum 5, 76131 Karlsruhe, Germany

\section{Author Contributions}

The manuscript was written through contributions of all authors. All authors have given approval to the final version of the manuscript. 


\section{Funding Sources \\ Helmholtz Society (program Science and Technology (STN)), Helmholtz Postdoc Programme (PD-157), Young Investigator Network (YIN)}

\section{Notes}

The authors declare no competing financial interest.

\section{ACKNOWLEDGMENT}

It is a pleasure to thank Sabessane Mounirattinam for help with geometry analysis of coiled lambda-shaped CNFs, Frank Winkler and Markus Guttmann for the preparation of the substrates, and Weibin $\mathrm{Wu}$ for helpful discussions. Furthermore, we thank the clean room team of the IMT for the kind manufacturing of the sample substrates. J. S. gratefully acknowledges funding from the Helmholtz Postdoc Programme (PD-157). S. M. acknowledges support by the Helmholtz Society through the program Science and Technology (STN). J. S. and M. H. thank the Young Investigator Network (YIN) for financial support through a YIN grant award. This work was partly carried out with the support of the Karlsruhe Nano Micro Facility (KNMF, www.kit.edu/knmf), a Helmholtz Research Infrastructure at Karlsruhe Institute of Technology (KIT, www.kit.edu).

\section{REFERENCES}

(1) Ando, Y.; Zhao, X.; Sugai, T.; Kumar, M. Growing Carbon Nanotubes. Mater. Today 2004, 7, 22-29.

(2) Zhang, M.; Li, J. Carbon Nanotube in Different Shapes. Mater. Today 2009, 12, 12-18.

(3) Feng, L.; Xie, N.; Zhong, J. Carbon Nanofibers and Their Composites: A Review of 
Synthesizing, Properties and Applications. Materials 2014, 7, 3919-3945.

(4) Rahman, G.; Najaf, Z.; Mehmood, A.; Bilal, S.; Shah, A.; Mian, S.; Ali, G. An Overview of the Recent Progress in the Synthesis and Applications of Carbon Nanotubes. C 2019, 5 , 3.

(5) Malik, S.; Nemoto, Y.; Guo, H.; Ariga, K.; Hill, J. P. Fabrication and Characterization of Branched Carbon Nanostructures. Beilstein J. Nanotechnol. 2016, 7, 1260-1266.

(6) Meng, G.; Han, F.; Zhao, X.; Chen, B.; Yang, D.; Liu, J.; Xu, Q.; Kong, M.; Zhu, X.; Jung, Y. J.; et al. A General Synthetic Approach to Interconnected Nanowire/Nanotube and Nanotube/Nanowire/Nanotube Heterojunctions with Branched Topology. Angew. Chemie - Int. Ed. 2009, 48, 7166-7170.

(7) Autumn, K. How Gecko Toes Stick. Am. Sci. 2006, 94, 124.

(8) Autumn, K.; Liang, Y. A.; Hsieh, S. T.; Zesch, W.; Chan, W. P.; Kenny, T. W.; Fearing, R.; Full, R. J. Adhesive Force of a Single Gecko Foot-Hair. Nature 2000, 405, 681-685.

(9) Autumn, K.; Sitti, M.; Liang, Y. A.; Peattie, A. M.; Hansen, W. R.; Sponberg, S.; Kenny, T. W.; Fearing, R.; Israelachvili, J. N.; Full, R. J. Evidence for van Der Waals Adhesion in Gecko Setae. Proc. Natl. Acad. Sci. U. S. A. 2002, 99, 12252-12256.

(10) Yurdumakan, B.; Raravikar, N. R.; Ajayan, P. M.; Dhinojwala, A. Synthetic Gecko FootHairs from Multiwalled Carbon Nanotubes. Chem. Commun. 2005, 3799.

(11) Zhao, Y.; Tong, T.; Delzeit, L.; Kashani, A.; Meyyappan, M.; Majumdar, A. Interfacial Energy and Strength of Multiwalled-Carbon-Nanotube-Based Dry Adhesive. J. Vac. Sci. 
Technol. B Microelectron. Nanom. Struct. 2006, 24, 331-335.

(12) Qu, L.; Dai, L.; Stone, M.; Xia, Z.; Wang, Z. L. Carbon Nanotube Arrays with Strong Shear Binding-On and Easy Normal Lifting-Off. Science 2008, 322, 238-242.

(13) Lutz, C.; Syurik, J.; Shyam Kumar, C. N.; Kübel, C.; Bruns, M.; Hölscher, H. Dry Adhesives from Carbon Nanofibers Grown in an Open Ethanol Flame. Beilstein J. Nanotechnol. 2017, 8, 2719-2728.

(14) Lutz, C.; Ma, Z.; Thelen, R.; Syurik, J.; Il'in, O.; Ageev, O.; Jouanne, P.; Hölscher, H. Analysis of Carbon Nanotube Arrays for Their Potential Use as Adhesives Under Harsh Conditions as in Space Technology. Tribol. Lett. 2019, 67, 10.

(15) Coelho, N. M. de A.; Furtado, J. L. B.; Pham-Huu, C.; Vieira, R. Carbon Nanofibers: A Versatile Catalytic Support. Mater. Res. 2008, 11, 353-357.

(16) Vieira, R. Carbon Nanofibers as Macro-Structured Catalytic Support. In Nanofibers; InTech, 2010; Vol. 395, pp. 116-124.

(17) AL-Hammadi, S. A.; Al-Amer, A. M.; Saleh, T. A. Alumina-Carbon Nanofiber Composite as a Support for MoCo Catalysts in Hydrodesulfurization Reactions. Chem. Eng. J. 2018, 345, 242-251.

(18) Ali, I.; Al-Arfaj, A. A.; Saleh, T. A. Carbon Nanofiber-Doped Zeolite as Support for Molybdenum Based Catalysts for Enhanced Hydrodesulfurization of Dibenzothiophene. J. Mol. Liq. 2020, 304, 112376.

(19) Zhou, D.; Seraphin, S. Complex Branching Phenomena in the Growth of Carbon 
Nanotubes. Chem. Phys. Lett. 1995, 238, 286-289.

(20) Li, J.; Papadopoulos, C.; Xu, J. Growing Y-Junction Carbon Nanotubes. Nature 1999, $402,253-254$.

(21) Papadopoulos, C.; Rakitin, A.; Li, J.; Vedeneev, A. S.; Xu, J. M. Electronic Transport in Y-Junction Carbon Nanotubes. Phys. Rev. Lett. 2000, 85, 3476-3479.

(22) Terrones, M.; Banhart, F.; Grobert, N.; Charlier, J.-C.; Terrones, H.; Ajayan, P. M. Molecular Junctions by Joining Single-Walled Carbon Nanotubes. Phys. Rev. Lett. 2002, 89,075505 .

(23) Gothard, N.; Daraio, C.; Gaillard, J.; Zidan, R.; Jin, S.; Rao, A. M. Controlled Growth of Y-Junction Nanotubes Using Ti-Doped Vapor Catalyst. Nano Lett. 2004, 4, 213-217.

(24) Meng, G.; Jung, Y. J.; Cao, A.; Vajtai, R.; Ajayan, P. M. Controlled Fabrication of Hierarchically Branched Nanopores, Nanotubes, and Nanowires. Proc. Natl. Acad. Sci. U. S. A. 2005, 102, 7074-7078.

(25) Choi, Y. C.; Choi, W. Synthesis of Y-Junction Single-Wall Carbon Nanotubes. Carbon N. Y. 2005, 43, 2737-2741.

(26) Heyning, O. T.; Bernier, P.; Glerup, M. A Low Cost Method for the Direct Synthesis of Highly Y-Branched Nanotubes. Chem. Phys. Lett. 2005, 409, 43-47.

(27) Liu, Q.; Liu, W.; Cui, Z.-M.; Song, W.-G.; Wan, L.-J. Synthesis and Characterization of 3D Double Branched K Junction Carbon Nanotubes and Nanorods. Carbon N. Y. 2007, 45, 268-273. 
(28) Ding, E.-X.; Wang, J.; Geng, H.-Z.; Wang, W.-Y.; Wang, Y.; Zhang, Z.-C.; Luo, Z.-J.; Yang, H.-J.; Zou, C.-X.; Kang, J.; et al. Y-Junction Carbon Nanocoils: Synthesis by Chemical Vapor Deposition and Formation Mechanism. Sci. Rep. 2015, 5, 11281.

(29) Lutz, C.; Bog, U.; Loritz, T.; Syurik, J.; Malik, S.; Kumar, C. N. S.; Kübel, C.; Bruns, M.; Greiner, C.; Hirtz, M.; et al. Locally Controlled Growth of Individual Lambda-Shaped Carbon Nanofibers. Small 2019, 15, 1803944.

(30) Shaikjee, A.; Coville, N. J. The Synthesis, Properties and Uses of Carbon Materials with Helical Morphology. J. Adv. Res. 2012, 3, 195-223.

(31) Raghubanshi, H.; Dikio, E. D. Synthesis of Helical Carbon Fibers and Related Materials: A Review on the Past and Recent Developments. Nanomaterials 2015, 5, 937-968.

(32) Raghubanshi, H.; Dikio, E. D.; Naidoo, E. B. The Properties and Applications of Helical Carbon Fibers and Related Materials: A Review. J. Ind. Eng. Chem. 2016, 44, 23-42.

(33) Krishna, V. M.; Somanathan, T.; Manikandan, E.; Umar, A.; Maaza, M. Large-Scale Synthesis of Coiled-like Shaped Carbon Nanotubes Using Bi-Metal Catalyst. Appl. Nanosci. 2018, 8, 105-113.

(34) Thakur, A.; Manna, A.; Samir, S.; Jindal, P. Polymer Nanocomposite Reinforced with Selectively Synthesized Coiled Carbon Nanofibers. Compos. Interfaces 2020, 27, 215226.

(35) Zhang, Q.; Yang, F.; Dong, H.; Yu, J.; Yu, L.; Dong, L. Application of SiO2 Spheres in the Synthesis of Coiled Carbon Nanofibers with High Purity. Diam. Relat. Mater. 2020, 
102,107664 .

(36) Zhao, Y.; Wang, J.; Huang, H.; Cong, T.; Yang, S.; Chen, H.; Qin, J.; Usman, M.; Fan, Z.;

Pan, L. Growth of Carbon Nanocoils by Porous $\alpha-\mathrm{Fe} 2 \mathrm{O} 3 / \mathrm{SnO} 2$ Catalyst and Its Buckypaper for High Efficient Adsorption. Nano-Micro Lett. 2020, 12, 1-17.

(37) Fu, X.; Pan, L.; Wang, Q.; Liu, C.; Sun, Y.; Asif, M.; Qin, J.; Huang, Y. Controlled Synthesis of Carbon Nanocoils on Monolayered Silica Spheres. Carbon N. Y. 2016, 99, $43-48$.

(38) Kumar, A.; Cross, A.; Manukyan, K.; Bhosale, R. R.; Van Den Broeke, L. J. P.; Miller, J. T.; Mukasyan, A. S.; Wolf, E. E. Combustion Synthesis of Copper-Nickel Catalysts for Hydrogen Production from Ethanol. Chem. Eng. J. 2015, 278, 46-54.

(39) Yudasaka, M.; Kikuchi, R.; Matsui, T.; Ohki, Y.; Yoshimura, S.; Ota, E. Specific Conditions for Ni Catalyzed Carbon Nanotube Growth by Chemical Vapor Deposition. Appl. Phys. Lett. 1995, 67, 2477-2479.

(40)

Qi, X.; Qin, C.; Zhong, W.; Au, C.; Ye, X.; Du, Y. Large-Scale Synthesis of Carbon Nanomaterials by Catalytic Chemical Vapor Deposition: A Review of the Effects of Synthesis Parameters and Magnetic Properties. Materials 2010, 3, 4142-4174.

(41) Ren, Z. F.; Huang, Z. P.; Xu, J. W.; Wang, J. H.; Bush, P.; Siegal, M. P.; Provencio, P. N. Synthesis of Large Arrays of Well-Aligned Carbon Nanotubes on Glass. Science 1998, $282,1105-1107$.

(42) Li, Y.-Y.; Hsieh, C.-C. Synthesis of Carbon Nanotubes by Combustion of a Paraffin Wax 
Candle. Micro Nano Lett. 2007, 2, 63.

(43) Hsieh, C. C.; Youh, M. J.; Wu, H. C.; Hsu, L. C.; Guo, J. C.; Li, Y. Y. Synthesis of Carbon Nanotubes Using a Butane-Air Bunsen Burner and the Resulting Field Emission Characteristics. J. Phys. Chem. C 2008, 112, 19224-19230.

(44) Pan, C.; Bao, Q. Well-Aligned Carbon Nanotubes from Ethanol Flame. J. Mater. Sci. Lett. 2002, 21, 1927-1929.

(45) Pan, C.; Liu, Y.; Cao, F.; Wang, J.; Ren, Y. Synthesis and Growth Mechanism of Carbon Nanotubes and Nanofibers from Ethanol Flames. Micron 2004, 35, 461-468.

(46) Bao, Q.; Pan, C. Electric Field Induced Growth of Well Aligned Carbon Nanotubes from Ethanol Flames. Nanotechnology 2006, 17, 1016-1021.

(47) Zhang, J.; Pan, C. Magnetic-Field-Controlled Alignment of Carbon Nanotubes from Flames and Its Growth Mechanism. J. Phys. Chem. C 2008, 112, 13470-13474.

(48) Wang, L.-J.; Li, C.-Z.; Gu, F.; Zhou, Q.-L. Morphology and Structure of Carbon Nanocoils Synthesized via the Flame Combustion of Ethanol. J. Inorg. Mater. 2008, 23, $1179-1183$.

(49) Choudhuri, A.; Camacho, J.; Chessa, J. Flame Synthesis of Coiled Carbon Nanotubes. Fullerenes Nanotub. Carbon Nanostructures 2006, 14, 93-100.

(50) Piner, R. D.; Zhu, J.; Xu, F.; Hong, S.; Mirkin, C. A. “Dip-Pen” Nanolithography. Science 1999, 283, 661-663.

(51) Liu, G.; Hirtz, M.; Fuchs, H.; Zheng, Z. Development of Dip-Pen Nanolithography (DPN) 
and Its Derivatives. Small 2019, 15, 1900564.

(52) Lutz, C. Bio-Inspired Dry Adhesives from Carbon Nanofibers and Their Potential Use in Space Technology, Karlsruhe Institute of Technology (KIT), 2018.

(53) Young, R. J. Carbon Fibre Composites: Deformation Micromechanics Analysed Using Raman Spectroscopy. In Structure and Multiscale Mechanics of Carbon Nanomaterials; Paris, O., Ed.; 2016; pp. 29-50.

(54) Davis, W. R.; Slawson, R. J.; Rigby, G. R. An Unusual Form of Carbon. Nature 1953, $171,756-756$.

(55) Fonseca, A.; Hernadi, K.; Nagy, J. B.; Lambin, P.; Lucas, A. A. Growth Mechanism of Coiled Carbon Nanotubes. Synth. Met. 1996, 77, 235-242.

(56) Szabó, A.; Fonseca, A.; Nagy, J. B.; Lambin, P.; Biró, L. P. Structural Origin of Coiling in Coiled Carbon Nanotubes. Carbon N. Y. 2005, 43, 1628-1633.

(57) Fejes, D.; Hernádi, K. A Review of the Properties and CVD Synthesis of Coiled Carbon Nanotubes. Materials 2010, 3, 2618-2642.

(58) Liu, L.; Zhao, J. Toroidal and Coiled Carbon Nanotubes. In Syntheses and Applications of Carbon Nanotubes and Their Composites; InTech, 2013; Vol. 395, pp. 116-124.

(59) Amelinckx, S.; Zhang, X. B.; Bernaerts, D.; Zhang, X. F.; Ivanov, V.; Nagy, J. B. A Formation Mechanism for Catalytically Grown Helix-Shaped Graphite Nanotubes. Science 1994, 265, 635-639.

(60) Pan, L.; Zhang, M.; Nakayama, Y. Growth Mechanism of Carbon Nanocoils. J. Appl. 
Phys. 2002, 91, 10058-10061.

(61) Zhong, D. Y.; Liu, S.; Wang, E. G. Patterned Growth of Coiled Carbon Nanotubes by a Template-Assisted Technique. Appl. Phys. Lett. 2003, 83, 4423-4425.

(62) Tang, N.; Wen, J.; Zhang, Y.; Liu, F.; Lin, K.; Du, Y. Helical Carbon Nanotubes: Catalytic Particle Size-Dependent Growth and Magnetic Properties. ACS Nano 2010, 4, $241-250$.

(63) Zhang, L.; Zhu, Y. B.; Ge, C. L.; Wei, C.; Wang, Q. L. The Synthesis of Carbon Coils Using Catalyst Arc Discharge in an Acetylene Atmosphere. Solid State Commun. 2007, $142,541-544$.

(64) Bandaru, P. R.; Daraio, C.; Yang, K.; Rao, A. M. A Plausible Mechanism for the Evolution of Helical Forms in Nanostructure Growth. J. Appl. Phys. 2007, 101.

(65) Tang, N.; Zhong, W.; Gedanken, A.; Du, Y. High Magnetization Helical Carbon Nanofibers Produced by Nanoparticle Catalysis. J. Phys. Chem. B 2006, 110, 1177211774.

(66) Tang, N.; Zhong, W.; Au, C.; Gedanken, A.; Yang, Y.; Du, Y. Large-Scale Synthesis, Annealing, Purification, and Magnetic Properties of Crystalline Helical Carbon Nanotubes with Symmetrical Structures. Adv. Funct. Mater. 2007, 17, 1542-1550.

(67) Loritz, T. Konstruktion, Aufbau Und Experimentelle Inbetriebnahme Einer Versuchsvorrichtung Zur Herstellung von Kohlenstoffnanoröhrchen, Karlsruhe Institute of Technology (KIT), 2017. 
Table of Content Graphics

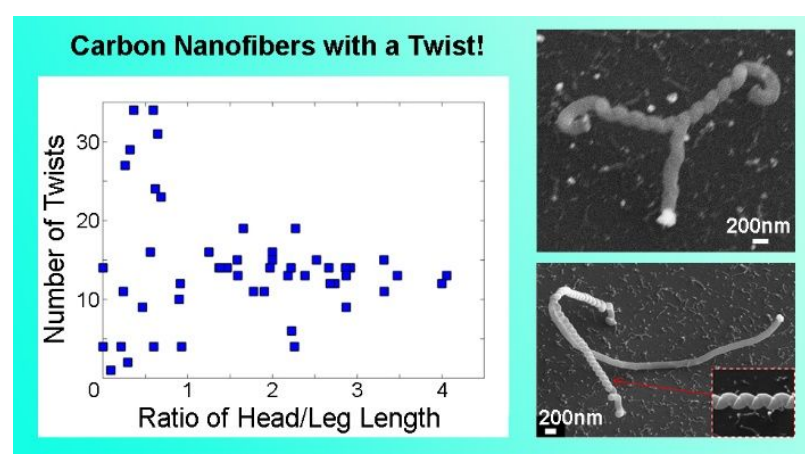

